Open Access

\title{
BASIC study: is intravaginal boric acid non-inferior to metronidazole in symptomatic bacterial vaginosis? Study protocol for a randomized controlled trial
}

\author{
Melinda Zeron Mullins ${ }^{*}$ and Konia M. Trouton
}

\begin{abstract}
Background: Bacterial vaginosis is associated with increased transmission of sexually transmitted infections, preterm labor, post-surgical infections, and endometritis. Current treatment for symptomatic bacterial vaginosis includes antibiotics, such as metronidazole, which are 70-80\% effective at one month after treatment and result in high recurrence rates and secondary candida infections. Intravaginal boric acid has been used for over a hundred years to treat vaginal infections, such as bacterial vaginosis. Boric acid is inexpensive, accessible, and has shown to be an effective treatment for other infections, such as vaginal candidiasis. To date, there has been no clinical trial evaluation of boric acid effectiveness to treat bacterial vaginosis.
\end{abstract}

Methods/Design: The BASIC (Boric Acid, Alternate Solution for Intravaginal Colonization) trial is a randomized, double-blinded, multicenter study. The study will enroll a minimum of 240 women of 16-50 years of age who are symptomatic with bacterial vaginosis. Eligible participants will have Amsel and Nugent scores confirming bacterial vaginosis. Women who are pregnant or menopausal or have other active co-infections will be excluded. Consenting participants who meet exclusion and inclusion criteria will be randomly assigned to one of three treatment groups: boric acid, metronidazole, or an inert placebo. Self-administration of treatment intravaginally for 10 days will be followed by clinical assessment at 7 and 30 days (days 17 and 40, respectively) after the end of the treatment phase. Primary outcome is a non-inferiority, per-protocol comparison of the effectiveness of boric acid with that of metronidazole at day 17, as measured by the Nugent score in 16-50 year olds. Secondary outcomes include: non-inferiority, intention-to-treat comparison of effectiveness of boric acid with that of metronidazole at day 17, analysis for both per-protocol and intention-to-treat at day 40 , and safety considerations, including adverse effects requiring patient discontinuation of treatment.

Discussion: This study will be the first to determine whether intravaginal boric acid is non-inferior to metronidazole in the treatment of bacterial vaginosis in symptomatic women.

Trial registration: ClinicalTrials.gov NCT00799214, registered online Nov 10, 2008.

Keywords: bacterial vaginosis, boric acid, double blinded, intravaginal, metronidazole, multicenter, non-inferiority, placebo, randomized controlled trial

\footnotetext{
* Correspondence: melindazeron@hotmail.com

Department of Family Practice, University of British Columbia, 3rd Floor David Strangway Building, 5950 University Boulevard, Vancouver, British Columbia BC V6T 1Z3, Canada
} 


\section{Background}

Bacterial vaginosis (BV) is thought to be more common than either vulvovaginal candidiasis or trichomoniasis, and to represent $40-50 \%$ of all cases of vaginitis [1]. Bacterial vaginosis is associated with postpartum endometritis, increased risk of post-surgical infections, pelvic inflammatory disease, increased risk of spontaneous abortions, preterm rupture of membranes and delivery, and increased risk of sexually transmitted infection acquisition, including: human immunodeficiency virus, herpes simplex virus, Neisseria gonorrhoeae, Chlamydia trachomatis, and Trichomonas vaginalis [1-6]. Bacterial vaginosis is caused by a shift from the normal peroxidaseproducing lactobacillus-dominant vaginal flora to a several-log increase in total bacterial colony count, which may include a mix of anaerobes, genital mycoplasmas, and Gardnerella vaginalis [1,7]. Bacterial vaginosis is classically characterized by abnormal odor and vaginal discharge in symptomatic women, while $50 \%$ of women with $\mathrm{BV}$ are asymptomatic $[7,8]$. It can also be painful, and is associated with dysuria or dyspareunia in symptomatic women [8]. Bacterial vaginosis is diagnosed in the presence of three of the four Amsel criteria: elevated $\mathrm{pH}$ $(>4.5)$; homogenous milky or creamy discharge; amine odor; and clue cells $[4,7,9]$. The gold standard for BV diagnosis is by Gram staining (Nugent score $\geq 7$ ), demonstrating a decrease or absence of lactobacilli and the presence of other microbes that cause BV $[4,7,9,10]$.

Untreated women show a $30 \%$ spontaneous resolution rate for $\mathrm{BV}[11,12]$. Current recommended antimicrobial treatments for BV in non-pregnant, symptomatic women include oral and intravaginal metronidazole or clindamycin $[1,8,13]$. Oral and intravaginal metronidazole are considered equivalent and effective treatments for BV $[9,11,14-16]$.

Standard treatment with metronidazole, according to these trials, has imperfect cure rates (approximately 70-80 \%) measured 1 month after treatment $[9,11,12,15-18]$ and high BV recurrence risks: $33 \%$ at 3 months [19] and approximately $49-66 \%$ at 1 year [18]. Metronidazole also carries a significant rate of side effects (10-20\% of women), especially with the oral metronidazole preparation [20], including secondary vaginal infection with candida $[12,14,16]$. At present, oral or vaginal metronidazole is the only recommended treatment option for recurrent $\mathrm{BV}$, as is vaginal metronidazole for long-term suppression $[9,21]$.

Boric acid $\left(\mathrm{H}_{3} \mathrm{BO}_{3}\right)$ has been used for over a hundred years for the treatment of vaginal infections and is commonly used by physicians and patients as an inexpensive, easy to use, accessible treatment of candidiasis and BV [22]. In addition to its proven effectiveness in the treatment of candidal infections, [22-31] including those that did not resolve with usual antifungal treatment [24-28], $\mathrm{H}_{3} \mathrm{BO}_{3}$ use seemed to be associated with a reduced number of co-infections with BV [25]. A retrospective study suggests clinical improvement in seven of nine patients following treatment with $600 \mathrm{mg}$ intravaginal $\mathrm{H}_{3} \mathrm{BO}_{3}$ for 14 nights in women with a mixed infection of $T$. glabrata vaginitis and BV [25]. In an uncontrolled, non-randomized, retrospective chart review, when $\mathrm{H}_{3} \mathrm{BO}_{3}$ was added to nitroimidazole there was promising long-term ( $>88 \%$ cure rate at 12 weeks after the study and $50 \%$ at 36 weeks of follow-up) suppression of recurrent BV [32]. Based on the aforementioned study, the physician evidence reference resource 'UpToDate [21] recommends that intravaginal $\mathrm{H}_{3} \mathrm{BO}_{3}(600 \mathrm{mg}$ for 21 days) be added to 7 days of metronidazole or tinidazole treatment to induce a remission prior to long-term suppression therapy. No studies to date have determined $\mathrm{H}_{3} \mathrm{BO}_{3}$ effectiveness in the treatment of acute, chronic, or recurrent $\mathrm{BV}$.

The objective of this study was to determine whether intravaginal $\mathrm{H}_{3} \mathrm{BO}_{3}$ is comparable to standard treatment, metronidazole, for the cure of BV in symptomatic women.

\section{Research question}

Among women $16-50$ years old symptomatic with $\mathrm{BV}$, is intravaginal treatment with $\mathrm{H}_{3} \mathrm{BO}_{3}$ non-inferior to metronidazole to achieve a Nugent score $<7$ (cure) by day 17 ?

\section{Null hypothesis}

$H_{0}$ : proportion of women cured using $\mathrm{H}_{3} \mathrm{BO}_{3} \leq$ metronidazole proportion of women cured using metronidazole $-10 \%$.

\section{Methods/Design Study design}

Institutional review board approval was granted for this study through the University of British Columbia Clinical Research Ethics Board (H07-02330). Health Canada Clinical Trial Application Notice of Authorization was obtained on 10 May 2013 (company code 31784, file number 187476). This trial is registered with ClinicalTrials.gov (NCT00799214). This study conforms to CONSORT 2010 guidelines for randomized control trials.

Based on preliminary data, the success rate for the boric acid treatment group is predicted to be approximately $77-88 \%[25,32]$ in treating BV. Metronidazole would be expected to be $70-80 \%[11,12,15-18]$ effective in treating $\mathrm{BV}$, as compared with a $30 \%$ success rate of a placebo group [11, 12]. The Nugent score is found to be $>95 \%$ sensitive [33] for detecting BV. We chose a non-inferiority limit of $10 \%$, as this will allow for a clinically relevant difference between the metronidazole treatment group and the boric acid group. Also, this is approximately $25 \%$ of the expected difference between 
results obtained for the metronidazole standard treatment group and the placebo group. Accounting for a non-inferiority limit (delta) of $10 \%$, testing for a significance level of 0.05 and power of $80 \%$, we require approximately 63 subjects per group. To allow for a dropout rate of $25 \%$, we set a target rate of 80 subjects per treatment arm. If there is a true difference in favor of the experimental treatment of $8 \%$, then 126 patients (total for two groups of 63) are needed, to be $80 \%$ sure of the upper limit of a one-sided $95 \%$ confidence interval, using a binary outcome, non-inferiority trial power calculator [34].

We felt it important to include a placebo treatment arm in this study for different reasons: (1) this is the first randomized controlled trial to use intravaginal $\mathrm{H}_{3} \mathrm{BO}_{3}$; thus, comparing it to placebo for safety and effectiveness would be of clinical interest (that is, perhaps it is not comparable to metronidazole in effectiveness but it is still significantly better than placebo), (2) having a placebo group in a non-inferiority trial acts as a sensitivity assay and internal validator for this type of trial [35], (3) comparing metronidazole effectiveness and safety with results obtained for a placebo group in 16-19-year-olds has not previously been done and would also be of clinical interest.

\section{Study setting}

Recruitment began February 2014 for a minimum of 240 volunteers through participating family practice clinics in British Columbia, Canada. Volunteer participating clinics represent a spectrum of different types of practice across British Columbia: rural, remote, urban, First Nations and non-First Nations communities, single and multi-clinician practices, general family practice offices, women's health clinics, and youth clinics. Clinics were recruited to the study through province-wide medical journal advertisements, university family practice departmental email advertisements, personal emails, faxes, phone calls and in person. Eligible clinics agreed to recruit five or more participants meeting inclusion and exclusion criteria and to use the study treatments and prepared packages provided.

\section{Participants and eligibility}

Women who present to their clinician at participating family practice clinics with symptoms of BV are offered an opportunity to participate in the study. Participants are given the informed consent form explaining the three possible treatment arms, and that participation will include three visits (the initial visit, day 0; day 17-19 [7-9 days after the treatment end to measure the primary endpoint]; and day 40-42 [30-32 days after the treatment end as a secondary endpoint]) involving three examinations, including a pregnancy test. There are no visits to the clinic while the participants are administering the 10-day treatment unless there are any concerns. Women are included in the study if they are symptomatic for BV, meet three of four Amsel criteria on the initial day of the study and subsequently have a swab graded with a Nugent score $\geq 7$. Detailed eligibility criteria are listed in Table 1 . In general, participants cannot be pregnant, menopausal, have another vaginal infection, or have an intrauterine device. Written consent is obtained prior to treatment intervention. Women who choose to participate receive their medication for free. There is a draw for recruited women to win a small gift certificate.

\section{Randomization}

Randomization in blocks of 80 was conducted by the study pharmacist (who was blinded to enrollment and outcome measurement) using the 'first generator' on the website: www.randomization.com in September 2013. These numbers then become the new identification numbers on the treatment packs. All treatment packs are then distributed to participating clinics by the study pharmacist who sends out five sequentially numbered treatment packs to each participating site as sites are recruited to the study. The study pharmacist is the only person with access to the master code to the randomized treatments. The participants, recruiting clinicians and investigators will be blinded to the controlled and randomized 10-day treatment pack that is given to the study participant. Blinded recruiting clinicians give out the physically indistinguishable treatment package in allocated randomization order to the sequential participants on enrollment day (day 0). Subsequently the treatment pack number becomes the participants' identification number.

\section{Intervention and control groups}

On day 0 , the enrolled participants will be blindly and randomly assigned to one of three 10-day treatments (minimum of 80 women per treatment arm): (1) placebo (emollient cream); (2) $\mathrm{H}_{3} \mathrm{BO}_{3}(600 \mathrm{mg}$ $\mathrm{H}_{3} \mathrm{BO}_{3}$ compounded in emollient cream); (3) metronidazole ( $10 \%$, for a total of $37.5 \mathrm{mg}$ metronidazole) intravaginal cream (Sanofi-Aventis Canada Inc. Product DIN 01926861) packaged by the study pharmacist. There is no detectable difference between the treatments in either appearance or scent.

\section{Study procedure and data collection}

On day 0 , a pregnancy test will be conducted and history will be collected by the clinician. During a pelvic examination, the clinician will ensure intact mucous membranes and anatomy, observe whether there is homogenous milky or creamy discharge (one of the four Amsel criteria), take swabs for chlamydia and gonorrhea, BV (for the 
Table 1 Eligibility criteria

\begin{tabular}{|c|c|}
\hline Inclusion criteria & Exclusion criteria \\
\hline $\begin{array}{l}\text { Must be } 16-50 \text { years old and pre-menopausal. Capable of giving written } \\
\text { informed consent. }\end{array}$ & Younger than 16 or post-menopausal. \\
\hline Fluent spoken and written English. & Menstruating at diagnosis. \\
\hline Agrees to examination on enrollment day, day $17-19$ and day $40-42$. & $\begin{array}{l}\text { Symptoms so severe as to make allocation to placebo unacceptable to } \\
\text { the woman. }\end{array}$ \\
\hline $\begin{array}{l}\text { Has a negative pregnancy test during the study. Agrees to follow study } \\
\text { protocol and is reliable for follow-up. }\end{array}$ & Currently pregnant or at high risk for pregnancy. \\
\hline $\begin{array}{l}\text { Has documented bacterial vaginosis infection by positive vaginal swab } \\
\text { (minimum Nugent score of } \geq 7 \text { ) and meets three of four Amsel criteria. }\end{array}$ & $\begin{array}{l}\text { Current active sexually transmitted infection (chlamydia, gonorrhea, } \\
\text { trichomonas). }\end{array}$ \\
\hline $\begin{array}{l}\text { Agrees to refrain from vaginal intercourse for the } 10 \text { days of treatment } \\
\text { (or to use non-lubricated non-latex condoms if unavoidable). Agrees not } \\
\text { to douche or use any intravaginal products during treatment (including } \\
\text { tampons, medications, devices). }\end{array}$ & $\begin{array}{l}\text { Current yeast infection as determined by history, physical examination, } \\
\text { and swab analysis. }\end{array}$ \\
\hline $\begin{array}{l}\text { Agrees to abstain from alcohol during the } 10 \text { days of treatment (from } \\
24 \text { hours before through } 72 \text { hours after taking study medication). }\end{array}$ & History of pelvic inflammatory disease. Allergy to metronidazole. \\
\hline Agrees to no new medications or antibiotics during treatment. & Currently breast feeding. \\
\hline \multirow[t]{6}{*}{$\begin{array}{l}\text { Has no currently active sexually transmitted infection as determined by } \\
\text { history, physical examination, and swab analysis negative for chlamydia, } \\
\text { gonorrhea, candidiasis, or trichomonas. }\end{array}$} & $\begin{array}{l}\text { Any open wound, excoriation, or vaginal irritation, including Bartholin's } \\
\text { cyst, abscess, and herpes simplex viral lesion, as determined by physical } \\
\text { exam. }\end{array}$ \\
\hline & $\begin{array}{l}\text { Presence of another vulvar, vaginal or medical condition, including } \\
\text { cervical neoplasia or treatment, or medical device, including intrauterine } \\
\text { device, that might confound treatment response. }\end{array}$ \\
\hline & Using lithium, anticoagulants or disulfiram drugs. \\
\hline & Any antifungal or antibiotic use 14 days prior to enrolment. \\
\hline & Papanicolaou smear taken within one week of enrolment. \\
\hline & Meeting three of four Amsel criteria but having a Nugent score $<7$. \\
\hline
\end{tabular}

Nugent score), candidiasis, and trichomonas, and perform a Whiff test using a provided standardized $10 \%$ potassium hydroxide solution (one of the four Amsel criteria). Vaginal discharge will also be tested for $\mathrm{pH}$ (one of the four Amsel criteria). Where facilities permit; clue cells will be examined, as the fourth Amsel criterion. On this day, the recruiting clinician will confirm participation, eligibility, and exclusion criteria using provided protocol forms and will collect the signed consent form and the demographic and medical history questionnaires provided.

The blinded treatment pack (containing either $\mathrm{H}_{3} \mathrm{BO}_{3}$ compounded in emollient cream, metronidazole cream, or emollient cream) is allocated by the recruiting clinician in the randomization order to sequential participants on day 0 . The participant package also contains: (1) vaginal applicator; (2) pads; (3) non-lubricated nonlatex condoms; (4) a diary to record daily use of the treatment (5) information sheet outlining follow-up instructions and emergency contact numbers for the study members. Participants will self-administer the blinded treatment cream intravaginally each evening immediately prior to sleep for 10 days. The participants will be instructed to use a provided pad during day and night.
Each participant will record daily compliance, side effects, and symptoms in the journal provided.

On both follow-up visits: days 17-19 (one week after the treatment end) and 40-42 (one month after the treatment end), the participant will return to the clinic that enrolled her for reassessment, a pregnancy test, and a follow-up examination, including a pelvic examination and repeat swab analysis. It will be noted whether her BV symptoms are still present, and if she had any side effects or problems during treatment. The participant will also return her daily treatment diary on the day 17 visit.

\section{Safety assessments}

Emergency telephone contact with the investigators will be available for any questions or problems related to the treatment. On day 5 of the treatment, the participant will receive a call, text, or email from one of the investigators to determine and record compliance, side effects, BV symptoms, and satisfaction with treatment. The study pharmacist will be informed by the recruiting clinician of the participant's full name, date of birth, and personal health number, and the study number assigned, so that the study pharmacist can enter the medication name into the British Columbia PharmaNet Database 
(the provincial pharmacy database). This safety measure ensures that subsequent treating physicians throughout the province will have access to details of the treatment received, in case of a safety concern and a need to unblind the participant's medication should the participant present with an emergency. Non-emergency access to the provincial pharmacy database is not allowed, to protect the participant's identification of the blinded treatment.

If at any time the participant reports intolerable side effects (vaginal discomfort, intolerable vaginal discharge, urticaria, or secondary yeast infection) during treatment, or exacerbation of symptoms at any point, she may voluntarily discontinue the treatment and leave the study without prejudice, and her doctor or another physician may provide treatment as per usual standard of care.

As neither metronidazole nor $\mathrm{H}_{3} \mathrm{BO}_{3}$ treatments provided to patients are in amounts considered toxic when taken intravaginally or if accidentally ingested, should there be any occurrence of serious adverse effects (neurological, cardiac, respiratory, gastrointestinal, or anaphylactic reactions) during any of the treatments, the study will be put on hold until an investigation can be held. In the case of serious adverse effects found to be caused by the treatment, the study will be stopped, and the study unblinded and decoded by the study pharmacist. The investigators, participants, and clinicians involved in the study would be informed for the reason for stopping the study.

\section{Outcomes and assessment}

On days $17-19$ and days $40-42$, if the vaginal swab is positive for BV found by both meeting three of four Amsel criteria and having a Nugent score $\geq 7$ and the participant is symptomatic for BV, the physician will prescribes a standard medication of his choice. This is considered a treatment failure addressing our primary and secondary outcomes: effectiveness at 7 days and 30 days after treatment end.

If the participant discontinued the treatment during the 10 days because of side effects or complained of intolerable side effects (major adverse effects) from the treatment, this is considered a treatment failure referring to our secondary outcome: safety.

\section{Statistical considerations}

The primary outcome measure is a non-inferiority perprotocol comparison of effectiveness of $\mathrm{H}_{3} \mathrm{BO}_{3}$ with metronidazole at day 17 , as measured by Nugent score, in 16-50-year-olds with a $z$-based confidence interval for the difference of two proportions. This was chosen as the primary outcome measure because the immediate effect of $\mathrm{H}_{3} \mathrm{BO}_{3}$ after a 10-day treatment compared with metronidazole was our most important question about BV treatment. A change from a baseline Nugent score $\geq 7$ to a
Nugent score $<7$ is considered a cure of BV. Treatment failure will be reported as a positive Nugent score $\geq 7$ at day 17.

Secondary outcome measures include a non-inferiority intention-to-treat comparison of effectiveness of $\mathrm{H}_{3} \mathrm{BO}_{3}$ with metronidazole at day 17 , as measured by Nugent score in 16-50-year-olds, with a $z$-based confidence interval for the difference of two proportions. We will use this comparison to confirm the robustness of our per-protocol comparison but the per-protocol analysis will be the primary determinant of the non-inferiority test. In addition, we will conduct a non-inferiority comparison of effectiveness of $\mathrm{H}_{3} \mathrm{BO}_{3}$ and metronidazole at day 40 , as measured by Nugent score in 16-50-year-olds analyzed on both per-protocol and intention-to treat principles, with a $z$-based confidence interval for the difference of two proportions, and safety consideration including intolerable adverse effects requiring patient discontinuation of the 10-day treatment. To control for multiple testing, we will use a Bonferroni-type correction. To account for both intention-to-treat and perprotocol analyses, and two timepoint analyses, the threshold for statistical significance will be set at 0.01 (approximately 0.05 divided by 4 ).

The following are tertiary outcome measures and no formal sample size calculations were made. Minor adverse effects (side effects not causing the patient to discontinue treatment) will be reported and compared between the treatment arms but will not be considered as treatment failure. We will examine the adolescent population, the 16-19 year old subgroup, for noninferiority for effectiveness of $\mathrm{H}_{3} \mathrm{BO}_{3}$, as compared with metronidazole, at the end of the study. Finally, we will test for superiority of the effectiveness and safety of $\mathrm{H}_{3} \mathrm{BO}_{3}$ compared with placebo in both the 16-50-yearolds and in the 16-19 year old subgroup. In addition, our study is the first to be able to examine 16-19-yearolds for the effectiveness and safety of metronidazole compared with placebo.

$95 \%$ confidence intervals will be established. Missing data (participants who fail to complete follow-up for any reason) are accounted for by an anticipated 25 \% lost-to-follow-up rate. Data will be blindly collected by the outcome assessors from the participating clinics and entered into an electronic database. The study pharmacist will provide the randomization code for the data to a third-party statistician and the data will be analyzed. Self-reported participant satisfaction criteria included at the end of the daily treatment diary will use a five-point rating scale to record: effectiveness in curing BV symptoms, side effects, ease of use, likelihood they would use it again for BV treatment, likelihood they would recommend it to someone else for BV treatment. 


\section{Discussion}

The BASIC (Boric Acid, Alternate Solution for Intravaginal Colonization) study will be the first prospective randomized control trial to determine the effectiveness of intravaginal $\mathrm{H}_{3} \mathrm{BO}_{3}$ compared with metronidazole for the treatment of $\mathrm{BV}$ in symptomatic women. If intravaginal $\mathrm{H}_{3} \mathrm{BO}_{3}$ is shown to be non-inferior to metronidazole in the treatment of $\mathrm{BV}$, this may provide women with less costly options for treating this common vaginal infection.

\section{Trial status}

The trial is currently open to recruitment.

\section{Abbreviations}

BASIC: Boric Acid, Alternate Solution for Intravaginal Colonization; BV: bacterial vaginosis.

\section{Competing interests}

The authors declare that they have no competing interests.

\section{Authors' contributions}

MZM is the principal investigator. MZM conceived and designed the study, prepared the initial draft of grant proposals, and wrote this manuscript, and will coordinate implementation and data acquisition. KT contributed to the conception and design and will assist with enrollment, data acquisition, and manuscript revision. Both authors approved the final manuscript.

\section{Authors' information}

MZM is a Clinic Instructor with the Department of Family Practice, University of British Columbia, working in Bella Coola, BC, Canada, and has a special interest in women's and adolescent health. KT is medical director of Vancouver Island Women's Clinic, Victoria, BC, Canada and is a Clinical Professor, with the Department of Family Practice, University of British Columbia.

\section{Acknowledgements}

Dr. Teresa Wood was involved in study conception and initial design and protocol development. Dr. Geoff Mullins helped to coordinate the study and reviewed the manuscript. Jeff Giles, the study pharmacist, assisted with the Health Canada application, and will assist with study coordination. Dr. Wendy $\vee$ Norman reviewed the manuscript. Dr. Jonathan Burkowitz, the study statistician, reviewed the initial design and the manuscript. MZM is supported during this study by the Clinical Scholar Program of the University of British Columbia. This study is supported by a generous research grant from the British Columbia College of Family Physicians Research Award 2013. No drugs, materials, or monetary funding or support are obtained from the industry sponsor or funder

Received: 21 January 2015 Accepted: 7 July 2015

Published online: 26 July 2015

\section{References}

1. Nyirjesy P, Mclntosh MJ, Steinmetz JI, Schumacher RJ, Joffrion JL. The effects of intravaginal clindamycin and metronidazole therapy on vaginal Mobiluncus morphotypes in patients with bacterial vaginosis. Sex Transm Dis. 2007:34(4):197-202.

2. Cherpes TL, Meyn LA, Krohn MA, Lurie JG, Hillier SL. Association between acquisition of herpes simplex virus type 2 in women and bacterial vaginosis. Clin Infect Dis. 2003;37:319-25.

3. Wiesenfeld HC, Hillier SL, Krohn MA, Landers DV, Sweet RL. Bacteria vaginosis is a strong predictor of Neisseria gonorrhoeae and Chlamydia trachomatis infection. Clin Infect Dis. 2003:36:663-8.

4. Yudin MH, Money DM, Infectious Diseases Committee. Screening and management of bacterial vaginosis in pregnancy. J Obstet Gynaecol Can. 2008;30(8):702-16.
5. Cohen CR, Lingappa JR, Baeten JM, Ngayo MO, Spiegel CA, Hong T, et al Bacterial vaginosis associated with increased risk of female-to-male HIV-1 transmission: a prospective cohort analysis among African couples. PLoS Med. 2012:9, e1001251.

6. Balkus JE, Richardson BA, Rabe LK, Taha TE, Mgodi N, Kasaro MP, et al. Bacterial vaginosis and the risk of Trichomonas vaginalis acquisition among HIV-1-negative women. Sex Transm Dis. 2014;41:123-8.

7. Gibbs RS. Asymptomatic bacterial vaginosis: is it time to treat? Am J Obs Gyne. 2007;196:495-6.

8. Anderson MR, Klink K, Cohrssen A. Evaluation of vaginal complaints. JAMA. 2004;291:1368-79.

9. Canadian STI Guidelines: Section 4. Management and treatment of specific syndromes: vaginal discharge (bacterial vaginosis, vulvovaginal candidiasis, trichomoniasis) 2013. http://www.phac-aspc.gc.ca/std-mts/ sti-its/cgsti-Idcits/section-4-8-eng.php. Accessed 20 May 2015.

10. Nugent RP, Krohn MA, Hillier SL. Reliability of diagnosing bacterial vaginosis is improved by a standardized method of Gram stain interpretation. J Clin Microbiol. 1991;29:297-301

11. Wain AM. Metronidazole vaginal cream $0.75 \%$ (MetroGel-Vaginal): a brief review. Inf Dis Obs Gyne. 1998;6:3-7.

12. Simoes JA, Bahamondes LG, Camargo RP, Alves VM, Zaneveld LJ, Waller DP, et al. A pilot clinical trial comparing an acid-buffering formulation (ACIDFORM gel) with metronidazole gel for the treatment of symptomatic bacterial vaginosis. Br J Clin Pharm. 2006;61:211-7.

13. Donders GG, Zodzika J, Rezeberga D. Treatment of bacterial vaginosis: what we have and what we miss. Expert Opin Pharmacother. 2014;15(5):645-57.

14. Ferris DG, Litaker MS, Woodward L, Mathis D, Hendrich J. Treatment of bacterial vaginosis: a comparison of oral metronidazole, metronidazole vaginal gel, and clindamycin vaginal cream. J Fam Pract. 1995;41:443-9.

15. Joesoef MR, Schmid GP, Hillier SL. Bacterial vaginosis: review of treatment options and potential clinical indications for therapy. Clin Inf Dis. 1999;28:S57-65.

16. Hay P. Bacterial vaginosis. Medicine. 2005;33:58-61.

17. Schmid GP. The epidemiology of bacterial vaginosis. Int J Gyne Obs 1999;67:S17-20.

18. Bradshaw CS, Morton AN, Hocking J, Garland SM, Morris MB, Moss LM, et al. High recurrence rates of bacterial vaginosis over the course of 12 months after oral metronidazole therapy and factors associated with recurrence. JID. 2006;193:1478-87.

19. Sobel JD, Ferris D, Schwebke J, Nyirjesy P, Wiesenfeld HC, Peipert J, et al. Suppressive antibacterial therapy with $0.75 \%$ metronidazole vaginal gel to prevent recurrent bacterial vaginosis. Am J Obs Gyne. 2006;194:1283-9.

20. Hanson JM, McGregor JA, Hillier SL, Eschenbach DA, Kreutner AK, Galask RP, et al. Metronidazole for bacterial vaginosis: a comparison of vaginal gel vs. oral therapy. J Reprod Med. 2000;45:889-96.

21. Sobel JD. Bacterial vaginosis. In: Post TW, editor. UpToDate ${ }^{\circ}$. Waltham, MA: Wolters Kluwer; 2015.

22. Keller Van Slyke K, Michel VP, Rein MF. Treatment of vulvovaginal candidiasis with boric acid powder. Am J Obs Gyne. 1981;141:145-8.

23. Swate TE, Weed JC. Boric acid treatment of vulvovaginal candidiasis. Obs Gyne. 1974;43:893-5.

24. Jovanovic R, Congema E, Nguyen HT. Antifungal agents vs boric acid for treating chronic mycotic vulvovaginitis. J Reprod Med. 1991;36:593-7.

25. Sobel JD, Chaim W. Treatment of Torulopsis glabrata vaginitis: retrospective review of boric acid therapy. Clin Inf Dis. 1997;24:649-52.

26. Guaschino S, De Seta F, Sartore A, Ricci G, De Santo D, Piccoli M, et al. Efficacy of maintenance therapy with topical boric acid in comparison with oral itraconazole in the treatment of recurrent vulvovaginal candidiasis. Am J Obs Gyne. 2001;184:598-602.

27. Sobel JD, Chaim W, Nagappan V, Leaman D. Treatment of vaginitis caused by Candida glabrata: use of topical boric acid and flucytosine. Am J Obs Gyne. 2003;189:1297-300

28. Ray D, Goswami R, Banerjee U, Dadhwal V, Goswami D, Mandal P, et al. Prevalence of Candida glabrata and its response to boric acid vaginal suppositories in comparison with oral fluconazole in patients with diabetes and vulvovaginal candidiasis. Diabetes Care. 2007;30(2):312-7.

29. Ray D, Goswami R, Dadhwal V, Goswami D, Banerjee U, Kochupillai N. Prolonged (3 month) mycological cure rate after boric acid suppositories in diabetic women with vulvovaginal candidiasis. J Infect. 2007;55(4):374-7.

30. Spence D. Candidiasis (vulvovaginal). BMJ Clin Evid. 2010;2010:0815.

31. Donders GG, Bellen G, Mendling W. Management of recurrent vulvo-vaginal candidosis as a chronic illness. Gyne Obs Invest. 2010;70(4):306-21. 
32. Reichman O, Akins R, Sobel JD. Boric acid addition to suppressive antimicrobial therapy for recurrent bacterial vaginosis. Sex Transm Dis. 2009:36(11):732-4.

33. Krohn MA, Hillier SL, Eschenbach DA. Comparison of methods for diagnosing bacterial vaginosis among pregnant women. J Clin Microbiol. 1989;27(6):1266-71.

34. sealed envelope ${ }^{\mathrm{TM}}$. Power (sample size) calculators. https:// www.sealedenvelope.com/power/binary-noninferior/. Accessed 3 June 2015.

35. Hida $\mathrm{E}$, Tango $\mathrm{T}$. Three-arm noninferiority trials with a prespecified margin for inference of the difference in the proportions of binary endpoints. J Biopharm Stat. 2013;23(4):774-89.

Submit your next manuscript to BioMed Central and take full advantage of:

- Convenient online submission

- Thorough peer review

- No space constraints or color figure charges

- Immediate publication on acceptance

- Inclusion in PubMed, CAS, Scopus and Google Scholar

- Research which is freely available for redistribution 